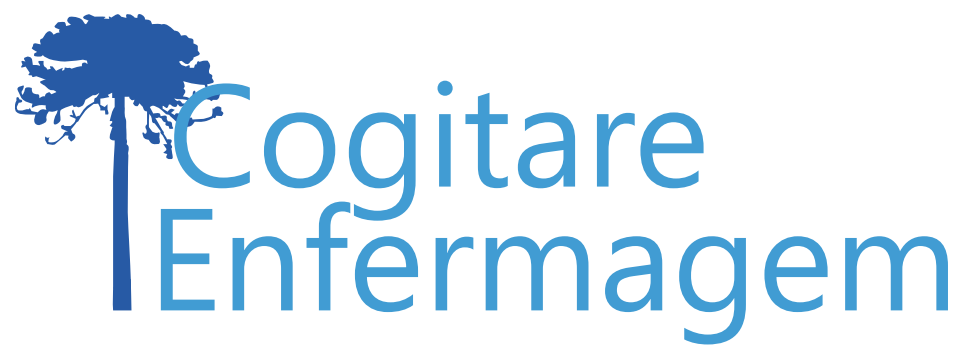

\title{
ADESÃO AO SEGUIMENTO CLÍNICO DE MULHERES PROFISSIONAIS DO SEXO COM SÍFILIS*
}

\begin{abstract}
Braulio Vieira de Sousa Borges', Elucir Gir², Marli Teresinha Gimenez Galvão³, Maria Eliete Batista Moura ${ }^{4}$, Giselle Mary Ibiapina Brito ${ }^{5}$, Rosilane de Lima Brito Magalhães ${ }^{6}$
\end{abstract}

\section{RESUMO}

Objetivo: avaliar a adesão ao seguimento clínico de mulheres profissionais do sexo com sífilis.

Metodologia: estudo transversal analítico, realizado na cidade de Teresina-PI, entre janeiro de 2016 a setembro de 2017. Utilizou-se método Respondent Driven Sampling para recrutamento e cupons válidos com informações sobre local e data das entrevistas. Foi considerada sífilis Venereal Disease Research Laboratory igual ou superior 1/1 sem tratamento prévio. Os dados foram analisados mediante teste Qui-quadrado de Pearson.

Resultados: a prevalência da sífilis nessas mulheres foi de 27 (7,5\%) e todas foram encaminhadas pelo pesquisador responsável para Unidades Básicas de Saúde. Desse total, oito $(29,6 \%)$ compareceram aos serviços de saúde, e quatro (14,8\%) realizaram tratamento adequado; nove (33,3\%) não fizeram adesão ao seguimento clínico e $10(37,0 \%)$ não foram localizadas após encaminhamento.

Conclusão: verificou-se elevada prevalência da sífilis, baixa adesão ao seguimento clínico e necessidade de melhor acolhimento em Unidades Básicas de Saúde.

DESCRITORES: Epidemiologia; Prevalência; Profissionais do Sexo; Sífilis; Cooperação e Adesão ao Tratamento.

\footnotetext{
*Artigo extraído da dissertação de mestrado "Adesão ao seguimento clínico de mulheres profissionais do sexo com sífilis". Universidade Federal do Piauí, 2017.
}

COMO REFERENCIAR ESTE ARTIGO: Borges BV de S, Gir E, Galvão MTG, Moura MEB, Brito GMI, Magalhães $\mathrm{R}$ de LB. Adesão ao seguimento clínico de mulheres profissionais do sexo com sífilis. Cogitare enferm. [Internet]. 2020 [acesso em "colocar data de acesso, dia, mês abreviado e ano"]; 25. Disponível em: http://dx.doi. org/10.5380/ce.v25i0.65456.

Este obra está licenciado com uma Licença Creative Commons Atribuição 4.0 Internacional.

${ }^{1}$ Enfermeiro. Mestre em Enfermagem. Enfermeiro da Rede de Atenção Básica do Distrito Federal. Brasília, DF, Brasil. $\odot$ ${ }^{2}$ Enfermeira. Doutora em Enfermagem. Docente de Enfermagem da Universidade de São Paulo. Ribeirão Preto, SP, Brasil. 9 ${ }^{3}$ Enfermeira. Doutora em Enfermagem. Docente de Enfermagem da Universidade Federal do Ceará. Fortaleza, CE, Brasil. 9 ${ }^{4}$ Enfermeira. Doutora em Enfermagem. Docente de Enfermagem da Universidade Federal do Piauí. Teresina, PI, Brasil. 9 ${ }^{5}$ Enfermeira. Mestre em Enfermagem. Universidade Federal do Paraná. Teresina, PI, Brasil. 9

${ }^{6}$ Enfermeira. Doutora em Enfermagem. Docente de Pós-Graduação em Enfermagem da Universidade Federal do Piauí. Teresina, PI, Brasil. 


\title{
ADHERENCE OF FEMALE SEX WORKERS WITH SYPHILIS TO CLINICAL FOLLOW-UP
}

\begin{abstract}
Objective: To evaluate the adherence of female sex workers with syphilis to clinical follow-up. Methodology: An analytical cross-sectional study was carried out in Teresina, a city in the state of Piauí, from January 2016 to September 2017. The respondent-driven sampling method was used for recruitment and valid coupons with information on the place and date of interviews. The Venereal Disease Research Laboratory test considered syphilis when equal to or higher than $1 / 1$ without previous treatment. The data were analyzed by application of Pearson's chisquared test.

Results: Of the study sample, 27 were diagnosed with syphilis (7.5\%), and they were all referred to primary healthcare units by the responsible researcher. Of these, eight $(29.6 \%)$ sought healthcare services and four (14.8\%) underwent appropriate treatment; nine (33.3\%) did not adhere to clinical follow-up; and ten (37.0\%) were not found after referral.

Conclusion: High syphilis prevalence was found, along with low adherence to clinical followup, and need for better care in primary healthcare units.
\end{abstract}

DESCRIPTORS: Epidemiology; Prevalence; Sex Workers; Syphilis; Treatment Adherence and Compliance.

\section{ADHESIÓN AL SEGUIMIENTO CLÍNICO DE TRABAJADORAS SEXUALES PROFESIONALES CON SIFILIS}

\author{
RESUMEN: \\ Objetivo: Evaluar la adhesión al seguimiento clínico de trabajadoras sexuales profesionales \\ con sífilis. \\ Metodología: Estudio transversal analítico, realizado en Teresina-PI, entre enero 2016 y \\ setiembre 2017. Se aplicó método Respondent Driven Sampling para reclutamiento y volantes \\ con información válida sobre lugar y fecha de las entrevistas. Se consideró sífilis Venereal \\ Disease Research Laboratory igual o superior a $1 / 1$ sin tratamiento previo. Datos analizados \\ mediante test de Chi-cuadrado de Pearson. \\ Resultados: La prevalencia de sífilis en dichas mujeres fue de 27 (7,5\%), todas fueron derivadas \\ por el investigador responsable a Unidades Básicas de Salud. De ese total, ocho $(29,6 \%)$ \\ comparecieron a los centros sanitarios; cuatro $(14,8 \%)$ se sometieron al tratamiento adecuado; \\ nueve $(33,3 \%)$ no adhirieron al seguimiento clínico, y $10(37,0 \%)$ no pudieron ser ubicadas \\ luego de ser derivadas. \\ Conclusión: Se verificó alta prevalencia de sífilis, escasa adhesión al seguimiento clínico y \\ necesidad de mejor acogimiento en Unidades Básicas de Salud.
}

DESCRIPTORES: Epidemiología; Prevalencia; Trabajadores Sexuales; Sífilis; Cumplimiento y Adherencia al Tratamiento. 
Estima-se que seis milhões de pessoas da população geral sejam infectadas por sífilis ao ano, no mundo(1). Em Mulheres Profissionais do Sexo (MPS), as taxas variam de $1,3 \%$ a $22,4 \%(2-3)$; dessa forma, são consideradas mais elevadas quando comparadas com a população em geral, variando de $4 \%$ a $13,3 \%{ }^{(4-5)}$.

Na Mongólia, na Ásia Oriental, a prevalência da sífilis também foi considerada elevada em MPS $(27,8 \%)$, entre os fatores associados destacam-se mulheres jovens com presença de úlcera genital(6). No México, estudo evidenciou MPS com sífilis com idade $\geq 30$ anos, com múltiplas parcerias, que haviam iniciado relações sexuais comerciais antes dos 20 anos de idade, usuárias de drogas ilícitas (cocaína, crack e metanfetaminas) ${ }^{(7)}$.

No Brasil também existe elevada prevalência de sífilis, dificuldades de rastreamento de casos e controle, acompanhamento do seguimento e possibilidades de resistência microbiana ao tratamento ${ }^{(8)}$. A prevalência da sífilis apresenta diferenças regionais para a população geral, sendo: 0,48\% (Sul), 0,73\% (Sudeste), 1,05\% (Norte), 1,14\% (Nordeste),

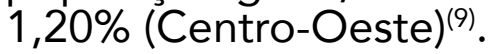

Nesse sentido, a sífilis é um grave problema de saúde pública no mundo, apresenta elevada prevalência em MPS, tem tratamento de baixo custo e difícil controle em diversas populações. Dessa forma, torna-se um fator de risco para aquisição pelo Vírus da Imunodeficiência Humana (HIV) e transmissão vertical. Assim, faz-se necessário um rastreamento precoce dos casos, acompanhamento do seguimento clínico e o tratamento para redução de novos agravos, a fim de reduzir a cadeia de transmissibilidade e a incidência da sífilis nessa população e nas parcerias sexuais.

Assim, MPS apresentam risco elevado para o HIV, considerando a ocorrência de outras Infecções Sexualmente Transmissíveis (IST) ${ }^{(10)}$. Elas apresentam maior vulnerabilidade individual quando fazem uso de drogas lícitas e ilícitas, uso inconsistente do preservativo associado a vulnerabilidades sociais e programáticas, como baixa escolaridade, acesso restrito aos serviços de saúde e estigmas sociais ${ }^{(11)}$.

O estudo teve como objetivo avaliar a adesão ao seguimento clínico de mulheres profissionais do sexo com sífilis.

\section{MÉTODO}

Estudo transversal do tipo descritivo sobre adesão ao seguimento clínico da sífilis com MPS, em Teresina-PI, entre janeiro de 2016 e setembro de 2017.

As participantes foram recrutadas pelo método Respondent Drive Sampling (RDS), a partir da seleção de sete sementes, com características diferentes em relação aos aspectos individuais e ao local de atuação, para possibilitar a composição de uma amostra mais representativa.

Em relação às características, optou-se por: semente 1: mulheres com atuação em praças; semente 2: mulheres com atuação em bares; semente 3: mulher jovem; semente 4: mulheres com atuação em boates: semente 5: mulher negra; semente 6: mulher adulta; e semente 7: mulher com atuação na rua. As sementes foram selecionadas com apoio da Associação de Prostitutas do Piauí - APROSPI, e justifica-se o método pelo fato dessa população ser organizada em rede e ter difícil acesso ${ }^{(10)}$.

Cada semente recebeu três cupons únicos, e não falsificáveis, e foi orientada para convidar mais três MPS de sua rede de atuação. E assim, cada participante elegível recebeu 
também mais três cupons até conseguir uma amostra significativa.

Estima que, em Teresina, há cerca de 600 MPS com atuação na prostituição. Assim, com base nessa informação e também em um estudo realizado com essa mesma população, que encontrou $402 \mathrm{MPS}^{(12)}$ e considerando uma prevalência de $4 \%$ de sífilis ${ }^{(13)}$, o erro tolerável de $2 \%$, nível de confiança $95 \%$, e o acréscimo de $10 \%$ em virtude de eventuais perdas, a amostra final foi 358 participantes.

Os critérios de inclusão foram: idade $\geq$ a 18 anos; atuar como MPS no município nos últimos quatro meses; ter tido pelo menos uma relação sexual mensal em troca de dinheiro nos últimos quatro meses; apresentar um cupom válido para participar do estudo. Exclusão: estar visivelmente sob a influência de drogas ilícitas, incluindo álcool, no momento da entrevista; e estar gestante, devido ao seguimento ambulatorial para gestantes ser mensal e, portanto, diferente da população geral.

Nesse processo, utilizou-se um formulário estruturado com as seguintes variáveis independentes: características sociodemográficas; comportamento sexual; adesão ao seguimento clínico e variável dependente: Venereal Disease Research Laboratory (VDRL) reagente, pela técnica de floculação.

Neste estudo, considerou-se caso de sífilis se: resultado de VDRL com titulação $\geq$ a $1 / 1$, sem tratamento prévio. E adesão ao seguimento clínico, o declínio da titulação em torno de duas diluições, após três meses do tratamento ${ }^{(8)}$. A diminuição dos títulos em torno de duas diluiçõ̃es indicou sucesso de tratamento e encerramento do caso(8). A coleta de dados ocorreu em locais específicos de atuação das MPS, ou seja, fechados (bares e boates) e abertos (praças e ruas), com data e horário definido por meio de um cupom válido e não falsificável.

O diagnóstico de sífilis foi informado de forma verbal e escrita, em formulário adequado para realização de encaminhamento para Unidade Básica de Saúde.

As participantes elegíveis foram submetidas à testagem rápida para detecção de sífilis. Para os resultados reagentes, foi investigado VDRL quantitativo. Após essa confirmação do diagnóstico, em média de sete dias, todas as MPS com titulação $\geq$ a $1 / 1$, sem tratamento prévio, foram encaminhadas para uma Unidade Básica de Saúde e agendado retorno, com intervalo de 30 dias, tendo como objetivo avaliar a adesão ao seguimento clínico. Após 60 dias, foi realizado novo VDRL para as MPS que buscaram o tratamento, para avaliar queda dos níveis de titulação.

Os dados foram digitados duplamente no Microsoft Excel em planilha eletrônica e após validação foram exportados para o software IBM® Statistical Package for the Social Sciences (SPSS) 21.0. Para a análise das variáveis quantitativas, foi apresentada a média, valores mínimos e máximos. Para verificar relações entre as variáveis, empregou-se o teste qui-quadrado, sendo considerada significância estatística em valor de $\mathrm{p}<0,005$.

A pesquisa foi aprovada pelo Comitê de Ética em Pesquisa da Universidade Federal do Piauí sob o parecer de número 0425.0.045.000-11.

\section{RESULTADOS}

Das 358 MPS, 241 (52,3\%) possuíam menos de oito anos de estudo, 19 (5,4\%) informaram ter parceria fixa fora da prostituição, 165 (46\%) declararam-se brancas. A renda mensal variou de sem rendimento a 10 salários mínimos, com média de um a dois em 147 $(41,1 \%)$. Um total de $301(84,1 \%)$ tinha de um a dois filhos, $136(38,0 \%)$ dessas mulheres possuíam atuação em locais abertos (ruas e praças) (Tabela 1). 
Tabela 1 - Distribuição do número e percentual de mulheres profissionais do sexo segundo aspectos sociodemográficos e prevalência da sífilis - 2016/2017. Teresina, PI, Brasil, 2017

$\begin{array}{lll}\text { Variáveis } & \mathrm{n}(\%) \quad \frac{\text { Prevalência da sífilis }(\mathrm{n}=358)}{\mathrm{n}(\%)} \quad \text { IC 95\%* }\end{array}$

Faixa etária (anos)

\begin{tabular}{lcccc}
\hline $18-24$ & $93(26)$ & $10(2,8)$ & & $(21,4-30,5)$ \\
\hline $25-39$ & $222(62)$ & $14(3,9)$ & 0,39 & $(56,9-67)$ \\
\hline $40-59$ & $43(12)$ & $3(0,8)$ & & $(8,6-15,3)$ \\
\hline Naturalidade & & & & \\
\hline Teresina & $277(77,4)$ & $23(6,4)$ & & $(73,0-81,7)$ \\
\hline Outro Local & $81(22,6)$ & $4(1,1)$ & 0,313 & $(18,2-26,9)$ \\
\hline
\end{tabular}

Ter companheiro fixo fora da prostituição

\begin{tabular}{lcccc}
\hline $\operatorname{Sim}$ & $19(5,4)$ & $1(0,3)$ & & $(3,0-7,7)$ \\
\hline Não & $339(94,6)$ & $26(7,2)$ & 0,057 & $(92,2-96,9)$ \\
\hline
\end{tabular}

Cor autorreferida

\begin{tabular}{|c|c|c|c|c|}
\hline Preta & $118(33)$ & $8(2,2)$ & & $(28,1-37,8)$ \\
\hline Branca & $165(46)$ & $9(2,5)$ & 0,001 & $(40,8-51,1)$ \\
\hline Outras & $75(21)$ & $10(2,8)$ & & $(16,7-25,2)$ \\
\hline \multicolumn{5}{|c|}{ Professa religião } \\
\hline Sim & $307(85,8)$ & $22(6,1)$ & & $(79,1-92,4)$ \\
\hline Não & $51(14,2)$ & $5(1,4)$ & 0,215 & $(10,5-17,8)$ \\
\hline \multicolumn{5}{|c|}{ Anos de estudo } \\
\hline Analfabeta & $20(5,6)$ & $1(0,2)$ & & $(3,2-7,9)$ \\
\hline 1 a 8 & $244(68,2)$ & $19(5,3)$ & 0,901 & $(63,3-73)$ \\
\hline$>8$ & $94(26,2)$ & $7(2)$ & & $(21,6-30,7)$ \\
\hline \multicolumn{5}{|c|}{ Renda mensal (salário mínimo) ${ }^{\star \star}$} \\
\hline$<1$ & $161(45)$ & $14(4)$ & 0,755 & $(39,8-50,1)$ \\
\hline 1 a 2 & $168(47)$ & $11(3)$ & & $(41,8-52,1)$ \\
\hline$\geq 3$ & $29(8)$ & $2(0,5)$ & & $(5,1-10,8)$ \\
\hline \multicolumn{5}{|c|}{ Número de filhos } \\
\hline Não tem & $200(56)$ & $9(2,5)$ & & $(50,8-61,1)$ \\
\hline 1 a 2 & $103(28,7)$ & $12(3,4)$ & 0,049 & $(24,0-33,3)$ \\
\hline$\geq 3$ & $55(15,3)$ & $6(1,6)$ & & $(11,5-19)$ \\
\hline \multicolumn{5}{|c|}{ Local de trabalho } \\
\hline Rua e praça & $136(38)$ & $13(3,6)$ & 0,241 & $(32,9-43)$ \\
\hline Bar e boate & $222(62)$ & $14(3,9)$ & & $(56,9-67)$ \\
\hline
\end{tabular}

Legenda: ${ }^{*} \mathrm{Cl}=$ Intervalo de Confiança de 95\%. ${ }^{\star * V a l o r ~ d o ~ s a l a ́ r i o ~ m i ́ n i m o ~ a ̀ ~ e ́ p o c a ~ d o ~ e s t u d o ~} \mathrm{R} \$ 788,00$.

Foi detectada uma prevalência de 7,5\% de sífilis de uma população de 358 MPS, 
com significância estatística em relação à cor ( $p \leq 0,001$, IC95\%=40,8-51,1), número de filhos ( $p \leq 0,049$, IC $95 \%=24,02-33,38)$ e prática sexual ( $p \leq 0,009$, IC $95 \%=82,41-89,59$ ) (Tabelas 1 e 2 ).

Tabela 2 - Distribuição do número e percentual de mulheres profissionais do sexo segundo o comportamento sexual e a prevalência da sífilis (2016/2017). Teresina, PI, Brasil, 2017

\begin{tabular}{|c|c|c|c|c|}
\hline \multirow[t]{2}{*}{ Variáveis } & \multirow[t]{2}{*}{$n(\%)$} & \multicolumn{2}{|c|}{ Prevalência da sífilis $(n=358)$} & \multirow[t]{2}{*}{ IC95\%* } \\
\hline & & $n(\%)$ & $\mathbf{P}$ & \\
\hline \multicolumn{5}{|l|}{ Comportamento sexual } \\
\hline Heterossexual & $340(94,9)$ & $25(6,9)$ & & $(92,6-97,1)$ \\
\hline Homossexual & $15(4,1)$ & $1(0,3)$ & 0,039 & $(2,0-6,1)$ \\
\hline Bissexual & $3(1)$ & $1(0,3)$ & & $(0-2)$ \\
\hline \multicolumn{5}{|c|}{ Parceria fixa remunerada na prostituição } \\
\hline Sim & $152(42,5)$ & $13(3,6)$ & & $(37,3-47,6)$ \\
\hline Não & $206(57,5)$ & $14(4)$ & 0,534 & $(52,3-62,6)$ \\
\hline \multicolumn{5}{|c|}{ Número de parceiros casuais na semana anterior à coleta } \\
\hline 1 a 5 & $106(29,6)$ & $8(2,2)$ & & $(24,8-34,3)$ \\
\hline 6 a 10 & $173(48,4)$ & $12(3,3)$ & 0,688 & $(43,2-53,5)$ \\
\hline 11 a 15 & $52(14,5)$ & $4(1,1)$ & & $(10,8-18,1)$ \\
\hline$>15$ & $27(7,5)$ & $3(0,9)$ & & $(4,7-10,2)$ \\
\hline \multicolumn{5}{|l|}{ Tipo de sexo } \\
\hline Vaginal & $308(86,0)$ & $19(5,3)$ & & $(82,4-89,5)$ \\
\hline Anal & $10(2,8)$ & $3(0,8)$ & 0,009 & $(1,1-4,5)$ \\
\hline Mais de um tipo & $40(11,2)$ & $5(1,4)$ & & $(7,9-14,4)$ \\
\hline \multicolumn{5}{|c|}{ Relação sexual no período menstrual } \\
\hline Sim, sem preservativo & $9(2,5)$ & $1(0,3)$ & & $(0,8-4,1)$ \\
\hline Sim, com preservativo & $61(17,0)$ & $6(1,7)$ & 0,68 & $(13,1-20,8)$ \\
\hline Não & $288(80,5)$ & $20(5,5)$ & & $(76,4-84,6)$ \\
\hline \multicolumn{5}{|c|}{ Uso de métodos contraceptivos } \\
\hline Sim & $107(29,8)$ & $10(2,8)$ & & $(25,0-34,5)$ \\
\hline Não & $251(70,2)$ & $17(4,7)$ & 0,405 & $(65,4-74,9)$ \\
\hline
\end{tabular}

Uso do preservativo masculino com o parceiro fixo na prostituição $(n=112)$

\begin{tabular}{|c|c|c|c|c|}
\hline Nunca usa & $91(25,5)$ & $10(2,8)$ & & $(20,9-30)$ \\
\hline Usa menos da metade das vezes & $21(5,8)$ & $2(0,5)$ & 0,3 & $(3,3-8,2)$ \\
\hline \multicolumn{5}{|c|}{ Uso do preservativo masculino com o parceiro casual } \\
\hline Sim & $285(79,6)$ & $25(7,0)$ & & $(75,4-83,7)$ \\
\hline Não & $69(19,2)$ & $2(0,5)$ & 0,215 & $(15,1-23,2)$ \\
\hline Às vezes & $4(1,2)$ & $0(0)$ & & $(0,1-2,3)$ \\
\hline
\end{tabular}

Legenda: ${ }^{\star} \mathrm{Cl}=$ Intervalo de Confiança de 95\% 
Nesse cenário, a respeito do comportamento sexual, 340 (94,9\%) referiram ser heterossexuais, e 152 (42,5\%) informaram ter parceiros fixos com remuneração na prostituição. O número de parceiros casuais na semana anterior à coleta variou de 1 a 15 clientes, sendo que 27 (7,5\%) tinham mais de 15 clientes.

A prática sexual vaginal foi relatada por $308(86,0 \%)$ mulheres, seguida por mais de um tipo de prática $40(11,2 \%)$. A prática sexual durante o período menstrual foi mencionada por 70 (19,5\%). Com relação ao uso de métodos contraceptivos, 107 (29,8\%) informaram usar algum método (Tabela 2).

Das 27 MPS com VDRL reagente, a primeira titulação variou de $1 / 1$ a 1/64 e todas as mulheres infectadas foram encaminhadas para tratamento. Desse total, oito $(29,6 \%)$ compareceram aos serviços de saúde pública e nove $(33,3 \%)$ não realizaram o seguimento clínico. Das mulheres que buscaram o serviço de saúde, foi detectada redução da titulação com variação de não reagente a $1 / 16$. Em relação ao tratamento recebido, sete $(25,9 \%)$ iniciaram após o primeiro atendimento, sendo que uma das mulheres $(3,7 \%)$ não recebeu gratuitamente o medicamento prescrito.

Das MPS que realizaram tratamento, seis $(22,2 \%)$ relataram ter parceria fixa na prostituição e fora dela. Em relação à comunicação do seu diagnóstico ao parceiro, quatro $(14,8 \%)$ informaram ao parceiro fixo fora da prostituição. Em relação ao uso do preservativo após o diagnóstico, somente quatro $(14,8 \%)$ relataram que sempre usam.

Em relação às condutas adotadas pelos profissionais de saúde, oito $(29,6 \%)$ das MPS informaram que não receberam solicitação para repetir o VDRL. No que se refere à orientação recebida durante o tratamento da sífilis, seis $(22,2 \%)$ receberam orientação sobre a importância de fazer o uso correto da medicação. No que tange à convocação das parcerias para fazer exames, somente dois $(7,4 \%)$ foram convocados.

\section{DISCUSSÃO}

A prevalência da sífilis em MPS foi de 7,5\% ( $n=27)$, considerada seis vezes mais elevada quando comparada com a prevalência da sífilis em gestantes de um estado da Região Nordeste do Brasi( ${ }^{(9)}$. Esse resultado tem semelhança com estudos desenvolvidos no mundo com MPS com sífilisi ${ }^{(3,6,13)}$.

Nesse sentido, prevalências mais elevadas da sífilis em MPS foram encontradas na Argentina e Mongólia, variando de $22,4 \%$ e $27,8 \%^{(6,14)}$. No cenário nacional, estudos evidenciaram prevalências que variaram de $4,0 \%$ a $13,3 \%(4-5)$.

Trata-se de uma população jovem e a maioria permanece durante todo o período reprodutivo na prostituição. Essas informações podem ser evidenciadas em outros estudos $^{(10-12)}$. A maior parte das mulheres era formada por adultas jovens e apresentaram prevalências mais elevadas quando comparadas com as demais. Esses achados também foram encontrados em estudos internacionais ${ }^{(13,15)}$.

A infecção por sífilis é motivo de preocupação, principalmente para as mulheres em idade reprodutiva, considerando a possibilidade de transmissão vertical. Conforme dados, no ano de 2013, a taxa de infecção para sífilis em gestantes do Piauí foi de 1,1\%(9).

Neste estudo, a prevalência da sífilis foi mais elevada em mulheres com baixa escolaridade e menor renda mensal, que não tinham companheiro. Na China, estudo com população semelhante mostrou que ter idade avançada, menor escolaridade, sexo comercial em casas de aluguel, ter mais clientes na semana anterior, sexo desprotegido e uso de drogas ilícitas, foram fatores associados à infecção por sífilis(16).

Neste estudo, houve associação significativa com a prevalência da sífilis em relação à 
cor da pele branca $(p \leq 0,001)$, número de filhos $(p \leq 0,049)$ e o tipo de sexo que pratica ( $p$ $\leq 0,009$ ); entretanto não foi identificado outro estudo com essa informação.

MPS atuam em ambientes abertos ou fechados com prevalências da sífilis mais elevadas em ambientes fechados. Um estudo mostrou que o número de parcerias sexuais, consumo de bebidas alcóolicas e drogas ilícitas é maior em ambientes fechados ${ }^{(17)}$. Isso sugere que MPS com atuação em ambientes fechados estão mais expostas à infecção por sífilis, considerando a multiplicidade de parcerias e a dificuldade de negociação do preservativo.

Quanto ao tipo de parceria, elas possuem parcerias fixas e casuais, na prostituição ou fora dela. Essa realidade também foi encontrada no cenário mundial ${ }^{(2,6)}$. Em relação ao uso do preservativo, esse estudo mostrou maior frequência com parceiro casual, comportamento evidenciado em diferentes regiões do mundo ${ }^{(18)}$.

Já na Etiópia, MPS relataram que o uso do preservativo ocorre em menos da metade das vezes, independentemente do tipo de cliente ${ }^{(18)}$. Neste estudo, mesmo diante da maior frequência do uso do preservativo com parceiro casual, foram encontradas prevalências mais elevadas de sífilis.

Os resultados do presente estudo mostram ainda que as MPS que não usam algum método contraceptivo apresentaram taxas mais elevadas de sífilis. Isso demonstra que fazer uso de um método contraceptivo pode se configurar em fator de proteção, considerando oportunidades para receber o preservativo e orientações para medidas de redução de danos. Na Argentina, o método contraceptivo mais popular entre MPS é o preservativo $(50,3 \%)$, seguido por comprimidos $(15,8 \%)$ e injeções $(11,8 \%)^{(14)}$.

A prática sexual vaginal foi referida com maior frequência e prevalência da sífilis, achado que vai ao encontro de outro estudo(14). A prática sexual anal entre MPS é mais rentável e o uso de preservativo é baixo por ser considerado inadequado para o sexo anal(19). Diante disso, torna-se imprescindível reconhecer que independentemente do tipo de prática sexual, a ausência de prevenção constituirá um fator de risco para aquisição de IST/HIV.

Em relação à adesão ao seguimento clínico das MPS, esta pesquisa apresentou níveis elevados de titulação pela técina de VDRL. As MPS apresentaram altas titulações. O VDRL, devidamente usado na rotina como teste único na maioria dos ambulatórios nacionais, apresenta alta concordância com os testes confirmatórios, considerando-se não somente os altos títulos 1/8, mas também os baixos títulos 1/1. Enfatiza-se que o VDRL, unicamente utilizado, representa um bom preditor da infecção sifilítica ${ }^{(20)}$.

Assim, destaca-se a necessidade de investimento em Unidades Básicas de Saúde para aumentar o rastreamento de casos de sífilis, e ainda melhor apoio laboratorial para realização de VDRL, uma vez que toda a conduta terapêutica dependerá inicialmente de um diagnóstico laboratorial.

Ao avaliar a adesão ao seguimento clínico, identificou-se queda na diminuição dos níveis da titulação das MPS que fizeram uso da medicação, bem como a completa negativação em metade dos casos. O sucesso no tratamento é identificado quando há diminuição dos títulos em torno de duas diluições em três meses, e três diluições em seis meses, após a conclusão do tratamento ${ }^{(8)}$. Dessa forma, reforça-se o tratamento correto para possibilidade de controle e eliminação da sífilis.

As MPS buscaram os serviços de saúde em tempo oportuno e com atendimento em menos de uma semana. No entanto, o retorno ao serviço de saúde foi baixo entre quase a totalidade das mulheres. As MPS só procuram a assistência à saúde quando se faz extremamente necessário, preferindo usar outros meios, como a automedicação, para aliviar seus problemas, antes de buscar as Unidades de Saúde ${ }^{(21)}$. Mediante os achados, é importante ampliar o acesso aos serviços de saúde com atendimento resolutivo e oportuno, a fim de evitar a perda de seguimento dos casos clínicos. 
Do total de mulheres encaminhadas nessa investigação, nove relataram dificuldades de acesso ao serviço de saúde, mesmo depois de novas tentativas. Autores reforçam que o estigma e a discriminação entre as MPS estão entre as principais barreiras ao acesso dos serviços de saúde ${ }^{(22)}$. Nesse sentido, faz-se necessário estimular a busca pelo serviço de saúde e melhor investimento em medidas de prevenção.

Nesta investigação, a comunicação da condição de saúde das MPS para as parcerias sexuais não ocorreu em todos os casos detectados. Um estudo mostrou que os principais fatores relacionados à não comunicação pela paciente-índice às parcerias sexuais foram: o medo e a insegurança ao revelar ao parceiro o diagnóstico, a cumplicidade e a preocupação com a saúde do parceiro, além da revelação do diagnóstico como forma de preservação do relacionamento ${ }^{(23)}$.

É importante que as parcerias sexuais sejam tratadas adequadamente, bem como promover ações que envolvam controle dos casos, notificação das doenças, busca ativa e o acompanhamento sorológico para a comprovação da cura dos $\operatorname{casos}^{(24)}$.

Com base nos relatos das MPS encaminhadas, neste estudo a abordagem às IST/HIV/ Síndrome da imunodeficiência adquirida e às Hepatites Virais entre MPS em Unidades de Saúde, não atendeu às necessidades dessa população, considerando falta de orientação adequada para o uso da medicação, importância quanto à adesão ao tratamento, falta de solicitação de outros exames e vacinação contra hepatite $B$.

O uso de testes rápidos é útil em situações de recursos limitados com acesso deficiente aos laboratórios ou rastreio para sífilis, refletindo no melhor acesso ao rastreio e tratamento dos casos de sífilis ${ }^{(25)}$. As ações para o controle da sífilis devem ser pautas das políticas públicas de saúde, com ênfase nas estratégias de rastreamento, diagnóstico e tratamento precoces, a fim de diminuir a morbidade e de elevar a melhoria da saúde sexual e reprodutiva da população geral e, em especial, das mais vulneráveis ${ }^{(26)}$.

Nessa perspectiva, o conhecimento sobre a temática é essencial à prevenção e adesão ao tratamento da doença, o que dificultará o processo de cura e prevençã̃o da enfermidade ${ }^{(27)}$. Para efetiva adesão ao tratamento clínico, faz-se necessária a assistência correta, não podendo ocorrer oportunidades perdidas.

No Protocolo Clínico e Diretrizes Terapêuticas para Atenção Integral às Pessoas com IST, recomenda-se aos profissionais de saúde que atendem pessoas com IST/HIV: oferta de preservativos e gel lubrificante, vacinação contra hepatite $B$ e contra o papiloma vírus humano, ênfase na adesão ao tratamento, informação e educação em saúde, convocação das parcerias sexuais, entre outras ${ }^{(8)}$.

Este estudo, realizado com uma população de difícil acesso e abordagem, com variáveis de cunho íntimo, pode ter causado constrangimentos e receios, minimizando ou ocultando algumas situações que influenciaram nos resultados, o que caracteriza uma possível limitação desta pesquisa.

\section{CONCLUSÃO}

Mulheres Profissionais do Sexo apresentam elevada prevalência da sífilis e baixa adesão ao seguimento clínico, demonstrando que há necessidade de melhor investimento no rastreamento de casos de sífilis e melhoria do acolhimento em Unidades Básicas de Saúde.

Pesquisas dessa natureza podem auxiliar para a maior visibilidade da problemática da sífilis e outros agravos em MPS e, dessa forma, contribuir para a implementação das políticas de saúde, considerando que trata-se de uma população de difícil acesso e que há necessidade de melhor investimento em atividades de educação em saúde, para garantir 
práticas sexuais mais seguras com vistas à redução de novos casos de IST.

\section{AGRADECIMENTOS}

\section{Este projeto foi financiado pelo Conselho Nacional de Desenvolvimento Científico e Tecnológico (CNPq 459935/2014-3).}

\section{REFERÊNCIAS}

1. Organización Mundial de la Salud (OMS). Proyectos de estrategias mundiales del sector de la salud. Infecciones de transmisión sexual, 2016-2021. Genebra: OMS; 2016. [acesso em 25 jan 2019. Disponível em: http://apps.who.int/gb/ebwha/pdf files/WHA69/A69 33-sp.pdf?ua=1.

2. Isac S, Ramesh BM, Rajaram S, Washington R, Bradley JE, Reza-Paul S, et al. Changes in HIV and syphilis prevalence among female sex workers from three serial cross-sectional surveys in Karnataka state, South India. BMJ Open [Internet]. 2015 [acesso em 25 jan 2019]; 5(3):e007106. Disponível em: http:// dx.doi.org/10.1136/bmjopen-2014-007106.

3. Zhu BY, Bu J, Huang PY, Zhou ZG, Yin YP, Chen XS, et al. Epidemiology os Sexually Transmitted Infections, HIV, and Related High Risk Behaviors among Female Sex Workers in Guangxi Automomous Region, China. Jpn J Infect Dis [Internet]. 2012 [acesso em 15 fev 2019]; 65(1):75-8. Disponível em: https://www.niid.go.jp/niid/images/JJID/65-1/75.pdf.

4. Pogetto MRB-Dal, Silva MG, Parada CMG de L. Prevalência de doenças sexualmente transmissíveis em mulheres profissionais do sexo, em um município do interior paulista, Brasil. Rev. Latino-Am. Enfermagem [Internet]. 2011 [acesso em 20 fev 2019]; 19(3):1-7. Disponível em: http://www.scielo.br/pdf/rlae/v19n3/ pt 07.pdf.

5. Szwarcwald CL. Taxas de prevalência de HIV e sífilis e conhecimento, atitudes e práticas de risco relacionadas às infecções sexualmente transmissíveis nos grupos das mulheres profissionais do sexo, no Brasil. Relatório técnico entregue ao Departamento de DST, Aids Hepatites Virais, 2009.

6. Munkhbaatar S, Aumakhan B, Jantsansengee B, Azyei I, Sanjaajamts Z, Badrakh J, et al. HIV and sexually transmitted infection-related risks among female sex workers in Mongolia: second-generation surveillance survey, 2011-2012. Sex Transm Infect [Internet]. 2014 [acesso em 25 fev 2019]; 90(6):463-8. Disponível em: http://dx.doi.org/10.1136/sextrans-2013-051443.

7. Bazzi AR, Rangel G, Martinez G, Ulibarri MD, Syvertsen JL, Bazzi SA, et al. Incidence and Predictors of HIV and Sexually Transmitted Infections Among Female Sex Workers and Their Intimate Male Partners in Northern Mexico: a longitudinal, multilevel study. Am J Epidemiol [Internet]. 2015 [acesso em 15 fev 2019]; 181(9):723-31. Disponível em: https://doi.org/10.1093/aje/kwu340.

8. Ministério da Saúde (BR). Protocolo Clínico e Diretrizes Terapêuticas. Atenção Integral às Pessoas com Infecções Sexualmente Transmissíveis. Protocolo Clínico e Diretrizes Terapêuticas para Atenção Integral às Pessoas com Infecções Sexualmente Transmissíveis Brasília: Ministério da Saúde; 2015. Disponível em: http://bvsms.saude.gov.br/bvs/publicacoes/protocolo clinico diretrizes terapeutica atencao integral pessoas infeccoes sexualmente transmissiveis.pdf.

9. Ministério da Saúde. Boletim Epidemiológico Sífilis 2015. Epidemiological Bulletin Syphilis. [Internt] $2015^{a} 2018$ [acesso em 20 fev 2019]. Disponível em: http://www.consultaesic.cgu.gov.br/busca/dados/ Lists/Pedido/Attachments/452305/RESPOSTA PEDIDO Boletim\%20Sifilis\%202015\%20final\%20-\%20 Cpia.pdf.

10. Szwarcwald CL, Damacena GN, Souza-Júnior PRB de, Guimarães MDC, Almeida W da S de, Ferreira AP de $\mathrm{S}$ et al. Factors associated with HIV infection among female sex workers in Brazil. 
Medicine [Internet]. 2018 [acesso em 20 fev 2019]; 97(1 Suppl); Disponível em: http://doi.org/10.1097/ MD.0000000000009013.

11. Patrício ACF de A, Ferreira MAM, Rodrigues BFL, Santos TD dos, Silva RAR da. Análise de conceito da vulnerabilidade ao HIV/aids em mulheres profissionais do sexo. Rev. Eletr. Enf. [Internet]. 2018 [acesso em 30 jul 2019]; 20:v20a38. Disponível em: https://doi.org/10.5216/ree.v20.49546.

12. Magalhães R de LB, Teles SA, Reis RK, Galvão MTG, Gir E. Low completion rate of hepatitis B vaccination in female sex workers. Rev. bras. enferm. [Internet]. 2017 [acesso em 20 jan 2019]; 70(3): 5149. Disponível em: http://dx.doi.org/10.1590/0034-7167-2016-0567.

13. Francis SC, Ao TT, Vanobberghen FM, Chilongani J, Hashim R, Andreasen A, et al. Epidemiology of curable sexually transmitted infections among women at increased risk for HIV in northwestern Tanzania: inadequacy of syndromic management. PLoS One [Internet]. 2014 [acesso em 15 fev 2019]; 9(7):e101221. Disponível em: https://doi.org/10.1371/journal.pone.0101221.

14. Pando M de los AP, Reynaga E, Coloccini RS, Fermepín MR, Kochel T, Montano SM, et al. Prevalencia de la infección por el VIH y de Treponema pallidum en trabajadoras sexuales de Argentina. Rev Panam Salud Publica [Internet]. 2011 [acesso em 25 fev 2019]; 30(4):303-8. Disponível em: https://www.scielosp. org/pdf/rpsp/2011.v30n4/303-308.

15. Remis RS, Kang L, Calzavara L, Pan Q, Liu J, Myers T, et al. Prevalence and correlates of HIV infection and sexually transmitted infections in female sex workers (FSWs) in Shanghai, China. Epidemiol Infect. [Internet]. 2015 [acesso em 15 fev 2019]; 143(2):258-66. Disponível em: https://doi.org/10.1017/ S0950268814000892.

16. Liu H, Dumenci L, Morisky DE, Xu Y, Li X, Jiang B. Syphilis among middle-aged female sex workers in China: a three-site cross-sectional study. BMJ Open [Internet]. 2016 [acesso em 25 fev 2019]; 6(5): e010420. Disponível em: http://dx.doi.org/10.1136/bmjopen-2015-010420.

17. Andrews $\mathrm{CH}$, Faxelid E, Sychaerun V, Phrasisombath K. Determinants of consistent condom use among female sex workers in Savannakhet, Lao PDR. BMC Womens Health [Internet]. 2015 [acesso em 20 jan 2019]; 19(15):63. Disponível em: https://doi.org/10.1186/s12905-015-0215-0.

18. Tamene MM, Tessema GA, Beyera GK. Condom utilization and sexual behavior of female sex workers in Northwest Ethiopia: a cross-sectional study. Pan Afr Med J [Internet]. 2015 [acesso em 15 fev 2019]; 21(50):1-10. Disponível em: http://doi.org/10.11604/pamj.2015.21.50.6009.

19. Wamoyi J, Mongi A, Sally M, Kakoko D, Shamba D, Geubbels E, et al. A qualitative study of discourses on heterosexual anal sexual practice among key, and general populations in Tanzania: implications for HIV prevention. BMC Public Health [Internet]. 2015 [acesso em 25 fev 2019]; 24(15):417. Disponível em: https://doi.org/10.1186/s12889-015-1768-4.

20. Campos JEB, Passos FDL, Lemos EA, Ferreira AW, Sá CAM, Silva LGP, et al. Significado laboratorial dos baixos títulos de VDRL para o diagnóstico da sífilis em gestantes, à luz das provas treponêmicas. DST j. bras. doenças sex. transm. [Internet]. 2008 [acesso em 28 jan 2019]; 20(1):12-7. Disponível em: http:// www.dst.uff.br/revista20-1-2008/2.pdf.

21. Leitão EF, Costa LLS, Brêda MZB, Albuquerque MC dos S, Jorge JS. A prática cotidiana de saúde das profissionais do sexo. Rev Bras Promoç. Saúde [Internet]. 2012 [acesso em 15 fev 2019]; 25(3):295-304. Disponível em: http://www.redalyc.org/articulo.oa?id=40823864007.

22. Folch C, Lazar C, Ferrer L, Sanclemente C, Casabona J. Female sex workers and access to social and health services in Catalonia: Influence of region of origin and place of work. AIDS Care [Internet]. 2013 [acesso em 25 fev 2019]; 25(08): 1033-8. Disponível em: https://doi.org/10.1080/09540121.2012.748872.

23. Cavalcante EGF, Miranda MCC, Carvalho AZFHT de, Lima ICV de, Galvão MTG. Partner notification for sexually transmitted infections and perception of notified partners. Rev. Esc. Enferm. USP [Internet]. 2016 [acesso em 15 fev 2019]; 50(03): 450-7. Disponível em: http://dx.doi.org/10.1590/S0080-

$\underline{623420160000400011 .}$ 
24. Oliveira DR de, Figueiredo MSN de. Abordagem conceitual sobre a sífilis na gestação e o tratamento de parceiros sexuais. Enferm. Foco [Internet]. 2011 [acesso em 15 fev 2019]; 2(2):108-11. Disponível em: https://doi.org/10.21675/2357-707X.2011.v2.n2.106.

25. Jafari Y, Peeling RW, Shivkumar S, Claessens C, Joseph L, Pai NP. Are treponema pallidum specific rapid and point-of-care test for syphilis accurate enough for screening in resourcer limited settingis? Evidence form a meta-analysis. Plos One [Internet]. 2013 [acesso em 20 jan 2019]; 8(2):e54695. Disponível em: https://doi.org/10.1371/journal.pone.0054695.

26. Pinto VM, Tancredi MV, Alencar HDR de, Camolesi E, Holcman MM, Grecco JP, et al. Prevalência de sífilis e fatores associados a população em situação de rua de São Paulo, Brasil, com utilização de teste rápido. Rev. bras. epidemiol. [Internet]. 2014 [acesso em 15 fev 2019]; 17(2):341-54. Disponível em: http:// dx.doi.org/10.1590/1809-4503201400020005ENG.

27. Vasconcelos MIO, Oliveira KMC de, Magalhães AHR, Guimarães RX, Linhares M do SC, Queiroz MVO, et al. Estratégias e desafios dos enfermeiros da atenção básica para o tratamento simultâneo da Sífilis. Atas Ciaiq. [Internet]. 2016 [acesso em 15 fev 2019]; 2(1): 1584-92. Disponível em: https://proceedings. ciaiq.org/index.php/ciaiq2016/article/view/918/902.

Recebido: 14/03/2019

Finalizado: 18/03/2020

Autor Correspondente:

Braulio Vieira de Sousa Borges

Universidade Federal do Piauí

CA 02, Bloco F, 229, Lago Norte, 71503-502, Brasília, DF, Brasil

E-mail: braulitos89@hotmail.com

Contribuição dos autores:

Contribuições substanciais para a concepção ou desenho do estudo; ou a aquisição, análise ou interpretação de dados do estudo - BVSB, RLBM

Elaboração e revisão crítica do conteúdo intelectual do estudo - BVSB, MEBM, GMIB

Aprovação da versão final do estudo a ser publicado - EG, MTGG

Responsável por todos os aspectos do estudo, assegurando as questões de precisão ou integridade de qualquer parte do estudo - RLBM 